\title{
A Tale of Two Towels
}

1Jyoti Sidhmalswamy Ghongdemath, ${ }^{2}$ Vishwanath Shindholimath, ${ }^{3}$ Krishna Lingegowda

\section{ABSTRACT}

Gossip about 'Gossypiboma' is still heard in the surgical field even in this decade too. The real incidence is unknown, but has been reported as 1 in 100 to 3000 for all surgical interventions; whereas it is 1 in 1000 to 1500 for intra-abdominal operations. We report two cases of 'retained surgical towel' after abdominal hysterectomy, to discuss the diagnosis, complications, management and propose various means of its prevention. Both the patients recovered well after a stormy postoperative period.

Keywords: Gossypiboma, Surgical towel, Hysterectomy, Retained sponge.

How to cite this article: Ghongdemath JS, Shindholimath V, Lingegowda K. A Tale of Two Towels. J South Asian Feder Obst Gynae 2014;6(1):41-45.

\section{Source of support: Nil}

\section{Conflict of interest: None}

\section{INTRODUCTION}

'Gossypiboma' is the technical term for an accidentally or unintentionally retained surgical sponge or towel inside the body of a patient following surgery. Wilson first described it in $1884 \mathrm{AD}$. The legal and medical issues have made the calculation of its exact incidence difficult. The incidence is one in 100 to 3000 for all surgeries; whereas it is 1 in 1000 to 1500 for abdominal surgeries. ${ }^{1}$ In this age of litigations against doctors for medical negligence, it is shocking to see such cases. With the quality of clinical care for patients becoming the predominant theme of this era, the need to redefine the standards of quality has never been this important. Here, we report two cases of gossypiboma to see its manifestations, complications, management and above all, methods to prevent it, an attempt to learn from others' mistakes.

\footnotetext{
${ }^{1}$ Assistant Professor, ${ }^{2}$ Associate Professor, ${ }^{3}$ Professor

1,3 Department of Obstetrics and Gynecology, PES Institute of Medical Sciences and Research, Kuppam, Andhra Pradesh India

${ }^{2}$ Department of Surgery, PES Institute of Medical Sciences and Research, Kuppam, Andhra Pradesh, India

Corresponding Author: Vishwanath Shindholimath, Associate Professor, Department of Surgery, PES Institute of Medical Sciences and Research, Kuppam, Andhra Pradesh, India e-mail: vishwashi@rediffmail.com
}

\section{CASE REPORTS}

\section{Case 1}

A 45-year-old woman presented with gradually progressive abdominal distension, vomiting and fever, 2 months after abdominal hysterectomy at a private nursing home, for dysfunctional uterine bleeding. On admission, she was febrile, pale and malnourished. Clinical examination revealed abdominal distension and tenderness over the previous scar. Laboratory investigations showed marked leukocytosis with neutrophilia (21,000 per cubic $\mathrm{mm})$. Ultrasonography revealed a large, mixed echogenic mass in the infraumbilical region. A provisional diagnosis of a retained sponge was made. At laparotomy, a large $25 \times 25 \mathrm{~cm}$, foul-smelling surgical towel, smeared with fecal matter, surrounded by small bowel mass was found (Fig. 1). It was very unusual to see such green colored surgical drape which was used as a surgical towel. Dense adhesions were found between the jejunal segment at $25 \mathrm{~cm}$ to the ligament of Treitz and the towel. The small bowel mesentery had completely surrounded the towel. Unhealthy fistulous small bowel was resected (see Fig. 1) and intestinal continuity was established by end-to-end anastomosis. Patient had prolonged ileus and sepsis for 10 days. She developed wound infection which was managed by dressing daily, was allowed orally on 10th postoperative day and subsequently discharged on the 18 th day. She did not report any complaints during followup and the clinical examination findings were normal, up to 12 months after discharge.

\section{Case 2}

A 40-years-old woman presented with fever, loose stool, loss of appetite and abdominal distension since 2 months, following abdominal hysterectomy 4 months back at a private nursing home, for dysfunctional uterine bleeding. She was admitted three times in the same hospital for the above complaints and was given antibiotics which did not relieve her. On examination, she was pale, malnourished and febrile. There was an ill-defined mass per abdomen extending above the umbilicus (Fig. 2) and tenderness over the previous scar. Laboratory investigations showed marked leukocytosis with neutrophilia (18000 per cubic mm). Ultrasonography revealed a large, echogenic mass with posterior shadowing in the pelvis. A provisional diagnosis of a retained sponge was made. At laparotomy, there were 

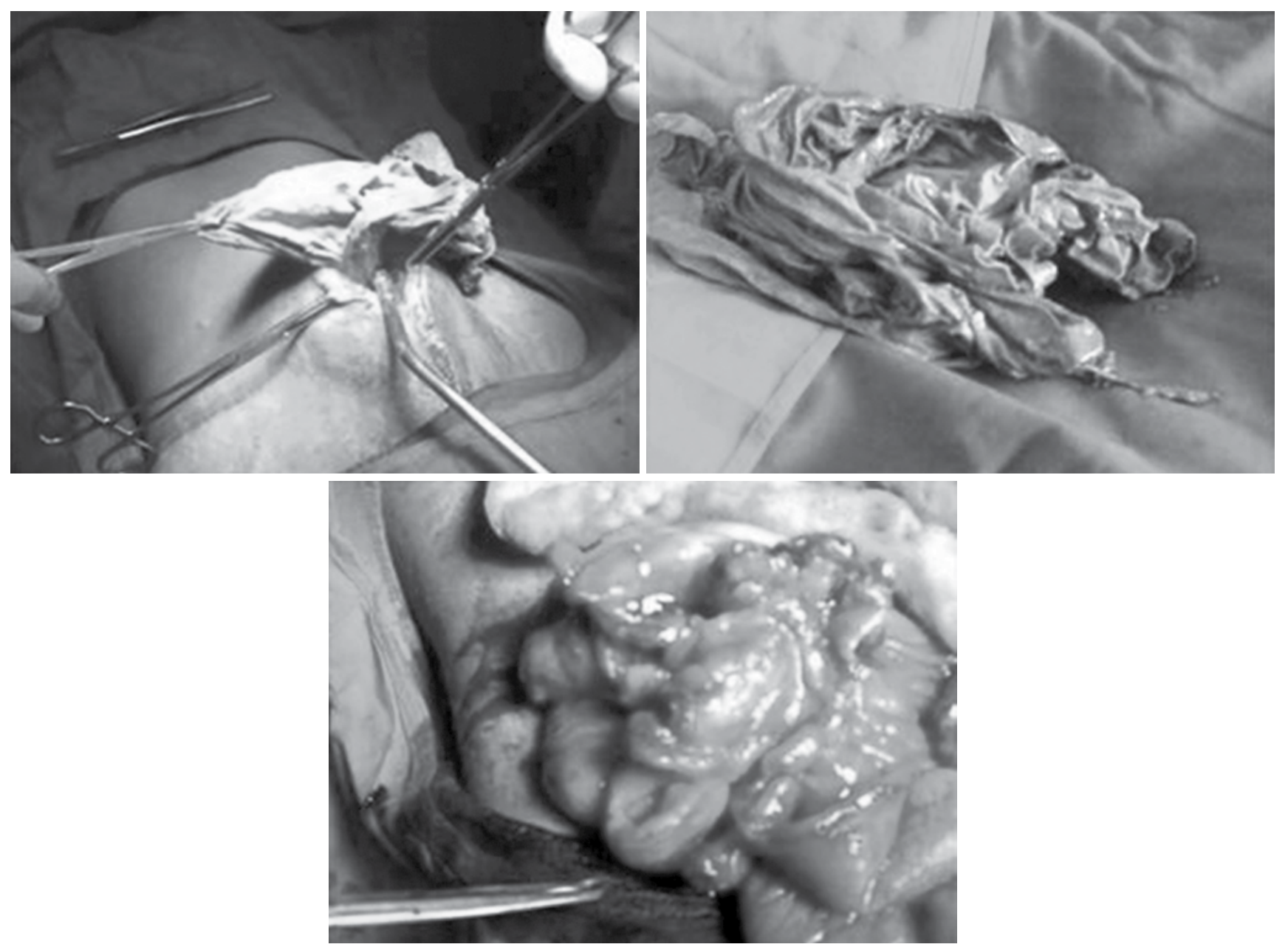

Fig. 1: Removal of retained intra-abdominal large, green, surgical towel and affected segment of small bowel

dense adhesions between the peritoneum and the bowel below the midline scar. A large $25 \times 20 \mathrm{~cm}$, foul-smelling surgical towel, smeared with fecal matter, surrounded by ileum was found (see Fig. 2). Surprisingly, the omentum was not involved in the mass formation. The ileum and the towel were densely adhered. The ileum was eroded by the towel at two places. The affected segment of the small bowel, $25 \mathrm{~cm}$ was resected (see Fig. 2) and intestinal continuity was established by end-to-end anastomosis. Skin was left open and secondary closure was done after 1 week. Patient had purulent discharge through abdominal drain for 7 days. She was allowed orally on seventh postoperative day and subsequently discharged on the 20th postoperative day. The patient had no complaints during follow-up and the clinical examination findings were normal, up to 10 months after discharge.

\section{DISCUSSION}

'Gossypiboma' is a foreign body made out of cotton, that is left unintentionally inside the patient, who undergoes an operation. Studies have shown that it occurs following various surgical procedures including abdominal, thoracic, orthopedic operations. ${ }^{2}$ This clinically inert, retained surgical sponge may serve as a locus for dreaded outcomes. Two types of pathological reactions can occur. ${ }^{3}$ One is 'Gossypiboma, an aseptic fibrinous type causing adhesions, granuloma or pseudo-tumor formation. ${ }^{1,2}$ The other, is an exudative type. Sometimes, the gossypiboma may remain unnoticed for ages, until the time it results in a complication or is found incidentally. The common complications are bowel obstruction, perforation, pseudotumor ${ }^{3-5}$ and granulomatous peritonitis. ${ }^{3}$ Usually, the body tries to expel such retained foreign bodies along the route of least resistance, either through the wound, sinus tract or rarely into intestine or vagina. Surgical sponge may erode an adjacent vessel-wall causing severe hemorrhage, during migration. As time passes, these foreign bodies may calcify or undergo complete resorption. ${ }^{4}$

Gossypiboma has to be considered in the differential diagnosis, when the patient does not have a timely recovery postoperatively. Previous surgical history gives clue to the diagnosis. If the patient presents with unusual symptoms or repeatedly visits with changing symptoms, thorough investigation is warrantied, including ultrasound and computed tomography (CT) scan. When symptomatic treatment fails, a second opinion from a senior colleague or 

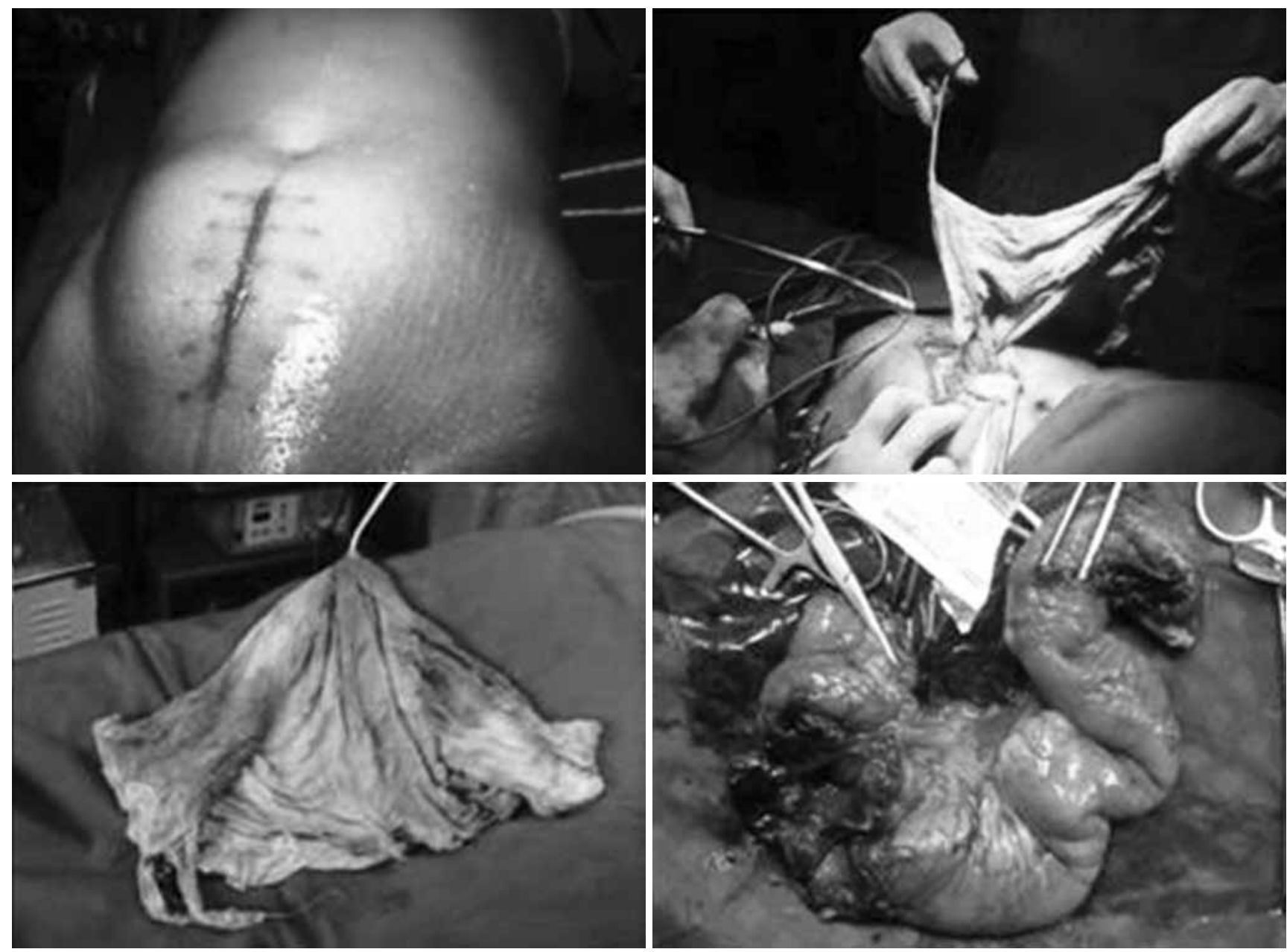

Fig. 2: Removal of retained large, white towel and resected segment of unhealthy fistulous small intestine

a radiologist helps. If necessary, a re-look laparotomy should not be hesitated, which might prove to be both diagnostic as well as therapeutic. Radiological findings of gossypibomas are variable. Plain abdominal radiography can diagnose it, when a radiopaque marker is seen. When these markers are disintegrated with passage of time, radiography won't aid in the diagnosis. ${ }^{5,6}$ Ultrasonography and CT scan are commonly used for the confirmation of the diagnosis. Ultrasound shows surgical sponges as brightly echogenic structure present in a cystic mass with acoustic shadowing posteriorly which changes with direction of the beam. CT scanning may show linear densities with spoke wheel configuration with gas trapped between the sponge fibers and cavity wall calcifications in chronic cases. Contrast enhancement of the rim may also be seen. ${ }^{2}$ Magnetic resonance imaging (MRI) shows a mass with variable signal intensity in accordance to the amount of fluid and protein in it. ${ }^{7}$

The risk factors for the retained surgical sponges can be patient-related, procedure-related or surgeon-related. Obese patients are at risk. More important are the procedure and surgeon related factors which include, emergency operations, unexpected change in the operative procedure, change in the operating team, long and difficult procedures which need to control blood loss by tamponade, multiple operations, ${ }^{3}$ pressure on the surgeon to curtail the operating time, other surgeries scheduled for that day at different places and last but not the least, a gossiping or a restless surgeon!

A stitch in time saves nine and a sponge removed on time keeps all fine. As soon as a gossypiboma is diagnosed, it needs to be taken off. Surgery was usually done to remove these foreign bodies since many years. But, few have reported alternative methods. Nosher and Siegel ${ }^{8}$ described six patients in whom percutaneous extraction was done successfully, where foreign bodies were removed, including an intra-abdominal laparotomy towel, two pelvic drains and an angiographic guide wire fragment; a superficially located sewing needle and bullet fragment were also removed. They propogated that, their new method can avoid long incisions, unnecessary dissection, the cost and risk of anesthesia. But, we have found that this method has got no value for removal of gossypibomas within the abdomen; the greater omentum, small and large bowel usually walling-off these retained sponges. Moreover, literature shows that, there may be dense adhesions between intra-abdominal organs and gossypibomas, at laparotomy. If the initial surgery had been performed a long time before the diagnosis of gossypiboma, there may be presence of any 


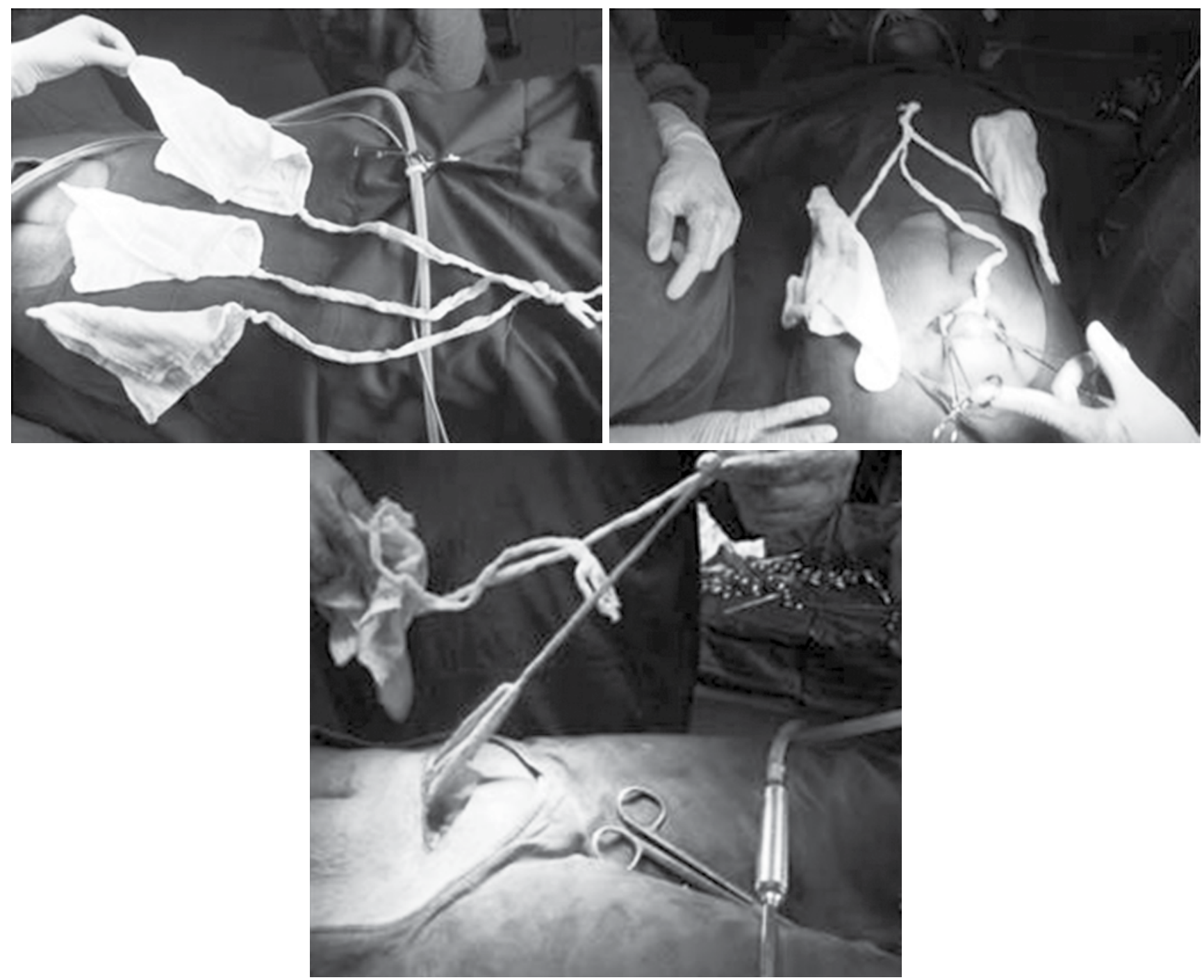

Fig. 3: Use of three sponges with long-tails tied into a knot as a preventive measure

fistula between the cavity containing the gossypiboma and the bowel, as was the case in the second patient. So, in such a situation, resection of the affected segment of the bowel becomes mandatory (see Figs 1 and 2).

To err each time and to learn is wrong. Prevention is the best policy. In the modern day, with all the advances in the surgical field and the hanging sword of consumer laws, the surgeon has to do justice and be as meticulous as ever, in each step of the operative procedure without any compromise. Try not to change the operating team often. The quality and safety are far more important than the speed of the procedure. Needless to say that haste makes waste. 'Once the specimen is out, the surgeon is out', should not be followed. The whole procedure 'skin-to-skin' is the primary responsibility of the operating surgeon and his team. Using large towels, as was done in above two cases, will not help, unless one is vigilant till the end of the surgery. The best way in prevention is to keep a count of surgical materials meticulously and thorough exploration of operative site at the end of the operative procedure. However, cases have been reported even when the pack count was normal, ${ }^{8}$ probably due to error in counting. Dhillon and Park ${ }^{9}$ also reported such a case, 11 months after hysterectomy; inspite of the count being performed twice as per the record. The routine use of surgical textile materials impregnated with radiopaque markers that are easily detected by intraoperative radiologic screening, also comes in handy when the count is suspicious. Another innovative aid would be to use a sponge with long tail, the tail lying outside on the abdomen, as a reminder, for easy identification and removal at the end of the surgery. If two or more sponges are needed, then their tails are tied together before use (Fig. 3) and at the end when one is pulled out, the rest follow, ensuring complete removal. It is advisable to adopt these towels with tail or else the towel may tell a different tale later. Better safe than sorry.

\section{CONCLUSION}

Although gossypiboma is rarely seen in day-to-day clinical practice, due to the rarity of the condition and at times, long latency in the manifestation of the symptoms, it frequently results in misdiagnosis leading to delay in management. But it should also be considered in the differential diagnosis of 
pain abdomen and abdominal distension in patients who have undergone laparotomy earlier. We suggest that:

1. Surgery should be performed in patients with abdominal gossypiboma, to remove the retained foreign body, if it is deeply located and/or a fistula is suspected.

2. The best approach in the prevention would be to keep a meticulous count of surgical materials and thorough exploration of the surgical site at the conclusion of surgery.

3. Routinely using surgical textile materials impregnated with a radiopaque marker help.

4. The use of sponge with a long tail, the tail kept outside, on the abdomen, that can be easily identified and removed at the end of the surgery, proves to be very practical.

\section{REFERENCES}

1. Rajagopal A, Martin J. Gossypiboma: 'a surgeon's legacy': report of a case and review of the literature. Dis Colon Rectum 2002;45:119-120.
2. Manzella A, Filho PB, Albuquerque E, Farias F, Kaercher J. Imaging of gossypibomas: Pictorial review. Am J Roentgenol 2009;193:94-101.

3. Gawande AA, Studdert DM, Orav EJ, Brennan TA, Zinner MJ. Risk factors for retained instruments and sponges after surgery. N Engl J Med 2003;348:229-235.

4. Kim CK, Park BK, Ha H. Gossypiboma in abdomen and pelvis: MRI findings in four patients. Am J Roentgenol 2007;189: 814817.

5. Dux M, Ganten M, Lubienski A, Grenacher L. Retained surgical sponge with migration into the duodenum and persistent duodenal fistula. Eur Radiol 2002;12:74-77.

6. Zbar AP, Agrawal A, Saeedi IT, Utidjian MRA. Gossypiboma revisited: a case report and review of the literature. JR Coll Surg Edinb 1998;43:417-418.

7. Asuquo ME, Ogbu N, Udosen J, et al. Acute abdomen from gossypiboma: a case series and review of literature. Nigerian J Surg Research 2006;8:174-176.

8. Nosher JL, Siegel R. Percutaneous retrieval of nonvascular foreign bodies. Radiology 1993;187:649-651.

9. Dhillon JS, Park A. Transmural migration of a retained laparotomy sponge. Am Surg 2002;68:603-605. 